Reprod. Nutr. Dévelop., 1988, 28 (1), 95-96.

\title{
Evaluation des coefficients d'utilisation digestive des constituants du triticale par le bélier adulte : comparaison de deux méthodes
}

\author{
P. LETERME, A. THÉWIS, E. THIRY
}

Faculté des Sciences Agronomiques de l'Etat, Service de Zootechnie Passage des Déportés, 2, B 5800 Gembloux, Belgique.

Summary. The regression method was compared with the difference method to determine the nutritive value of triticale in sheep. More accurate results were obtained with the regression method and with the difference method when the ration included at least $60 \%$ of triticale.

Vu les difficultés inhérentes à la détermination des coefficients d'utilisation digestive d'un concentré chez le ruminant (Berge, 1982 ; Giger et Sauvant, 1983), nous avons entrepris de déterminer ceux du triticale en comparant la méthode des différences à celle par régression.

Matériel et méthodes. Seize béliers Texel adultes, répartis en 4 lots, reçoivent un régime composé de foin de prairie hâché, de triticale aplati (variété Clercal) et de minéraux $(50 \mathrm{~g} / \mathrm{j})$. Le triticale représente $0,30,60$ ou $80 \%$ de la matière sèche (MS) ingérée. Les animaux font l'objet de 2 bilans successifs : d'abord $\left(B_{1}\right)$ avec un foin de qualité médiocre distribué en quantité limitée $\left(40 \mathrm{~g} \mathrm{MS} / \mathrm{kg} \mathrm{P}^{0,75}\right)$; puis $\left(B_{2}\right)$ avec un foin de qualité moyenne $\left(50\right.$ à $\left.60 \mathrm{~g} \mathrm{MS} / \mathrm{kg} \mathrm{P}^{0,75}\right)$. Les aliments, les refus et les fèces sont analysés selon le schéma classique de Weende. La composition chimique des foins (en $\%$ de la MS) est la suivante : $B_{1}: 91,4 \%$ de matière organique (MO), $8,2 \%$ de matières azotées totales (MAT) et $32,3 \%$ de cellulośse brute (CB) ; $B_{2}: 90,4 \%$ de MO, 9,8\% de MAT et $31,1 \%$ de CB ; celle du triticale est de $98,4 \%$ de MO, $11,9 \%$ de MAT et $3,3 \%$ de CB.

Résultats et discussion. Lors des deux bilans, l'augmentation de la proportion de triticale dans la ration induit un accroissement hautement significatif de la dMO et des matières azotées digestibles (MAD en \% de la MS) (tabl. 1). Pour $B_{1}$, les relations entre la proportion du principe alimentaire considéré du triticale $(C)$ et de 
la dMO ou les MAD de la ration sont linéaires. Pour $B_{2}$, par contre, l'ajustement mathématique met en évidence un phénomène d'interaction négative entre le triticale et le foin. Dans ce cas, les valeurs de dMO et MAD extrapolées pour le triticale seul ne présentent guère d'autre intérêt que de permettre de chiffrer l'importance des interactions digestives dans le cas de rations mixtes. L'interaction négative observée pour la dMO s'explique sans doute par l'antagonisme bien connu entre la digestion des glucides fermentescibles et celle des parois végétales, antagonisme amplifié par le niveau alimentaire plus élevé dans $B_{2}$ que dans $B_{1}$. En effet, I'augmentation du niveau de triticale dans la ration a provoqué une chute plus rapide de la dCB de la ration dans $B_{2}$ que dans $B_{1}$ (tabl. 1). Dans notre cas, l'analyse des erreurs attachées aux calculs établit que les meilleures estimations de la dMO et des MAD sont obtenues à la fois par régression et par différence lorsque le régime comprend au moins $60 \%$ de triticale (tabl. 1). Mais bien que les erreurs calculées ne soient pas significativement différentes lorsque $\mathrm{C}=60 \%$ ou $80 \%$, c'est néanmoins pour ce dernier niveau de triticale qu'elles sont les plus faibles, ce qui confirme les résultats de Berge (1982) et Giger et Sauvant (1983). Concernant les MAD du triticale, il faut souligner les teneurs anormalement faibles obtenues. Ceci est dû aux faibles concentrations en MAT des rations (10\% environ).

TABL. 1. - Influence de la proportion de triticale (T) sur la digestibilité de la MO (dMO), de la CB (dCB) et la teneur en MAD (en \% de la MS) de la ration: dMO et MAD du triticale calculées par régression $(\mathrm{R})$ et par différence $(\mathrm{D})$; erreurs attachées aux calculs de digestibilité du triticale.

\begin{tabular}{|c|c|c|c|c|c|c|c|c|c|c|c|c|c|}
\hline & & & & & & \multicolumn{4}{|c|}{ Triticale } & \multicolumn{4}{|c|}{ Erreurs (*) } \\
\hline & & \multicolumn{4}{|c|}{ Ration } & $R$ & \multicolumn{3}{|c|}{$\mathrm{D}$} & $R$ & \multicolumn{3}{|c|}{$\mathrm{D}$} \\
\hline & $\mathrm{T}$ & 0 & 30 & 60 & 80 & & 30 & 60 & 80 & & 30 & 60 & 80 \\
\hline $\mathrm{B}_{1}$ & $\begin{array}{l}\mathrm{n} \\
\mathrm{dMO} \\
\mathrm{MAD} \\
\mathrm{dCB}\end{array}$ & $\begin{array}{r}3 \\
54,9^{a} \\
3,7^{a} \\
51,9^{a}\end{array}$ & $\begin{array}{r}4 \\
63,7^{\mathrm{b}} \\
5,2^{\mathrm{b}} \\
47,0^{\mathrm{a}}\end{array}$ & $\begin{array}{r}3 \\
74,6^{\mathrm{c}} \\
6,4^{\mathrm{c}} \\
47,3^{\mathrm{a}}\end{array}$ & $\begin{array}{c}4 \\
79,1^{d} \\
6,8^{d} \\
35,3^{b}\end{array}$ & $\begin{array}{r}84,7 \\
7,4\end{array}$ & $\begin{array}{r}83,5^{\mathrm{e}} \\
7,7^{\mathrm{e}}\end{array}$ & $\begin{array}{r}87,5^{\mathrm{e}} \\
7,7^{\mathrm{e}}\end{array}$ & $\begin{array}{r}83,9^{e} \\
7,3^{e}\end{array}$ & $\begin{array}{l}2,3^{x} \\
0,3^{x}\end{array}$ & $\begin{array}{l}8,7^{y} \\
0,5^{y}\end{array}$ & $\begin{array}{l}4,8^{x} \\
0,2^{x}\end{array}$ & $\begin{array}{l}2,3^{x} \\
0,4^{x}\end{array}$ \\
\hline $\mathrm{B}_{2}$ & $\begin{array}{l}\text { n } \\
\text { dMO } \\
\text { MAD } \\
\text { dCB }\end{array}$ & $\begin{array}{r}3 \\
61,5^{\mathrm{a}} \\
5,4^{\mathrm{a}} \\
58,6^{\mathrm{a}}\end{array}$ & $\begin{array}{r}4 \\
65,2^{b} \\
6,0^{b} \\
44,8^{b}\end{array}$ & $\begin{array}{r}3 \\
69,9^{c} \\
6,1^{c} \\
34,0^{b}\end{array}$ & $\begin{array}{c}2 \\
80,2^{\mathrm{d}} \\
7,0^{\mathrm{d}} \\
38,6^{\mathrm{c}}\end{array}$ & $\begin{array}{r}87,8 \\
7,4\end{array}$ & $\begin{array}{r}73,5^{\mathrm{e}} \\
7,2^{\mathrm{e}}\end{array}$ & $\begin{array}{r}75,9 e \\
6,5^{e}\end{array}$ & $\begin{array}{r}84,3^{f} \\
7,2^{\mathrm{e}}\end{array}$ & $\begin{array}{l}1,6^{x} \\
0,3^{x}\end{array}$ & $\begin{array}{l}6,6 \\
0,6\end{array}$ & $\begin{array}{l}1,6^{x} \\
0,3^{x}\end{array}$ & $\begin{array}{l}1,1^{x} \\
0,1^{x}\end{array}$ \\
\hline
\end{tabular}

a, b, c, d: $\alpha<0,05 ;$ e,f : $\alpha<0,05 ; \mathrm{x}, \mathrm{y}:(\chi<0,05$.

$\left(^{*}\right)$ Erreurs $: \mathrm{D}:$ odT $=$ odRation $/ \mathrm{C}+(1 / \mathrm{C}-1)$. odfoin.

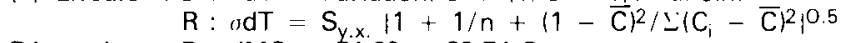

Régressions : $\mathrm{B}_{1}: \mathrm{dMO}=54,99+29,74 \mathrm{C}$

MAD $=3,77+3,64 \mathrm{C}$

$\mathrm{B}_{2}: \mathrm{dMO}=61,91+25,84 \mathrm{C}^{2}$

MAD $=5,58+1,78 \mathrm{C}^{2}$

$\begin{array}{ll}r=0,978^{* * *} & S_{y_{y . x}}=1,99 \\ r=0,981^{* * *} & S_{y . x .}=0,23 \\ r=0,978^{* * *} & S_{y . x .}=1,37 \\ r=0,890^{* * * *} & S_{y . x .}=0,23\end{array}$

En conclusion, la méthode par régression et celle des différences lorsque la ration comprend au moins $60 \%$ de triticale, permettent toutes deux d'estimer la digestibilité du triticale avec une bonne précision. Cependant, seule la méthode par régression permet de mettre en évidence une interaction digestive.

Berge Ph., 1982. Th. Dr Ing. Université de Montpellier, 105 pages.

Giger S., Sauvant D., 1983. Ann. Zootechn., 32, 215-246. 